\title{
Effectiveness of a pathway-driven eHealth- based integrated care model (PEICM) for community-based hypertension management in China: study protocol for a randomized controlled trial
}

Zheyu Wang ${ }^{1+}$, Chengling $\mathrm{Li}^{2+}$, Wencai Huang ${ }^{3+}$, Yan Chen ${ }^{1}$, Yuqiong $\mathrm{Li}^{2}$, Libin Huang ${ }^{3}$, Mei Zhang ${ }^{2}$, Dan Wu', Li Wang ${ }^{3}$, Huilong Duan', Jiye An ${ }^{1}$ and Ning Deng ${ }^{1 *}$ (D)

\begin{abstract}
Background: The prevalence of hypertension is high and increasing in China in recent years. The treatment and control of hypertension calls for long-term management beyond hospital, which is hard to implement in traditional care settings. Integrated care combined with information technology can promote high-quality healthcare services across the life-course. However, few studies have applied a customized integrated care model in community-based hypertension management in China, catering to the emerging "three-manager" mode. This study aims to identify the effectiveness of a pathway-driven eHealth-based integrated model that implemented as a full-featured telehealth system to facilitate standardized management of hypertension in China.
\end{abstract}

Methods: The trial has been designed as a 1-year, non-blinded superiority trial with two parallel groups. A total of 402 hypertensive patients who meet the eligibility criteria will be recruited and randomized with a 1:1 allocation. All the participants will receive a mobile device for self-management, which is a part of our telehealth system.

Participants in the control group will only use the device for BP measurement and receive regular follow-ups from care providers according to the guidelines. Participants in the intervention group will gain full access to the system and receive intervention based on the proposed model (a well-designed coordinated care pathway consisting of 9 tasks). Outcomes will be measured mainly on three occasions (at inclusion, at 6 months, and at 12 months). The primary outcome is mean change in systolic blood pressure over a 12-month period. Secondary outcomes include changes in diastolic blood pressure, biochemical indexes related to hypertension, lifestyles, self-management adherence, and hypertension awareness, as well as work efficiency of care providers.

(Continued on next page)

\footnotetext{
* Correspondence: zju.dengning@gmail.com

'Zheyu Wang, Chengling Li and Wencai Huang contributed equally to this work.

${ }^{1}$ College of Biomedical Engineering and Instrument Science, The Ministry of Education Key Laboratory of Biomedical Engineering, Zhejiang University, Hangzhou 310027, China

Full list of author information is available at the end of the article
}

(c) The Author(s). 2021 Open Access This article is licensed under a Creative Commons Attribution 4.0 International License, which permits use, sharing, adaptation, distribution and reproduction in any medium or format, as long as you give appropriate credit to the original author(s) and the source, provide a link to the Creative Commons licence, and indicate if changes were made. The images or other third party material in this article are included in the article's Creative Commons licence, unless indicated otherwise in a credit line to the material. If material is not included in the article's Creative Commons licence and your intended use is not permitted by statutory regulation or exceeds the permitted use, you will need to obtain permission directly from the copyright holder. To view a copy of this licence, visit http://creativecommons.org/licenses/by/4.0/ The Creative Commons Public Domain Dedication waiver (http://creativecommons.org/publicdomain/zero/1.0/) applies to the data made available in this article, unless otherwise stated in a credit line to the data. 
(Continued from previous page)

Discussion: This study aims to investigate whether a pathway-driven eHealth-based integrated care model based on the "three-manager" mode will improve hypertension control in China. Success of the model would help improve the quality of present community-based management procedures and benefit more patients with uncontrolled hypertension.

Trial registration: Chinese Clinical Trial Registry ChiCTR1900027645. Registered on November 22, 2019.

Keywords: Hypertension management, Integrated care, Pathway, Community, Telehealth, Study protocol, Randomized controlled trial

\section{Administrative information}

Note: the numbers in curly brackets in this protocol refer to Additional file 1: SPIRIT checklist item numbers. The order of the items has been modified to group similar items (see http://www.equator-network.org/reporting-guidelines/ spirit-2013-statement-defining-standard-protocol-items-forclinical-trials/).

\begin{tabular}{|c|c|}
\hline Title $\{1\}$ & $\begin{array}{l}\text { Effectiveness of a pathway-driveneHealth- } \\
\text { based integrated care model (PEICM) for } \\
\text { community-based hypertension manage- } \\
\text { ment in China: study protocol for a ran- } \\
\text { domized controlled trial }\end{array}$ \\
\hline Trial registration $\{2 \mathrm{a}$ and $2 \mathrm{~b}\}$. & $\begin{array}{l}\text { Chinese Clinical Trial Registry } \\
\text { ChiCTR1900027645, registered on } \\
\text { November 22, } 2019 \text {. }\end{array}$ \\
\hline Protocol version $\{3\}$ & $\begin{array}{l}\text { Issue Date: } 7 \text { Nov } 2019 \\
\text { Protocol Version: Original } \\
\text { This manuscript details the protocol on } \\
\text { the original version approved on } 7 \\
\text { November } 2019 .\end{array}$ \\
\hline Funding $\{4\}$ & $\begin{array}{l}\text { This study was supported by the } \\
\text { National Key Research and Development } \\
\text { Program of China (No.2017YFC0114105, } \\
\text { No.2018YFC0910503, } \\
\text { No.2017YFB1002301), the Key Research } \\
\text { and Development Program of Ningxia } \\
\text { Hui Autonomous of China (No. } \\
\text { 2018BFG02009). }\end{array}$ \\
\hline Author details $\{5 a\}$ & $\begin{array}{l}{ }^{1} \text { College of Biomedical Engineering and } \\
\text { Instrument Science, The Ministry of } \\
\text { Education Key Laboratory of Biomedical } \\
\text { Engineering, Zhejiang University, } \\
\text { Hangzhou, China } \\
{ }^{2} \text { The First People's Hospital of Yibin, } \\
\text { Yibin, China } \\
{ }^{3} \text { ZICT Technology Co., Ltd., Shenzhen, } \\
\text { China }\end{array}$ \\
\hline $\begin{array}{l}\text { Name and contact } \\
\text { information for the trial } \\
\text { sponsor }\{5 b\}\end{array}$ & $\begin{array}{l}\text { Trial Sponsor: The National Key } \\
\text { Research and Development Program of } \\
\text { China \& The Key Research and } \\
\text { Development Program of Ningxia Hui } \\
\text { Autonomous of China } \\
\text { Contact name: Haoming Li } \\
\text { Email: dengn@zju.edu.cn }\end{array}$ \\
\hline Role of sponsor $\{5 c\}$ & $\begin{array}{l}\text { The funding body had no role in study } \\
\text { design and will not have any role } \\
\text { during collection, management, analysis } \\
\text { and interpretation of data; writing of } \\
\text { the manuscript; and the decision to } \\
\text { submit results. }\end{array}$ \\
\hline
\end{tabular}

\section{Introduction}

\section{Background and rationale $\{6 \mathrm{a}\}$}

Hypertension is a major risk factor for cardiovascular disease and affects more than $20 \%$ of the adult population in China, according to the latest national survey [1]. The treatment and control of hypertension calls for long-term patient self-management along with supervision and intervention from doctors [2]. However, hypertension management within the traditional care settings is disconnected and time-consuming, which cannot meet long-term care needs of patients [3]. Integrated care is proposed as an approach to transform health services to meet these challenges [4]. In an integrated care setting, health services are delivered by a coordinated multidisciplinary team of providers, aiming to promote the comprehensive delivery of quality services across the life-course [5].

The advent of information technology facilitates the delivery of integrated care services and has demonstrated potential to improve the outcome of hypertension management [6-8]. The importance of information technology in integrated care can be interpreted from different scales. From an individual scale, care planning is a central approach of integrated care, which aims to deliver more personalized and targeted care by creating shared care plans. The care plans clearly articulate the role of each provider and patient in the care process [9]. The use of information technology is considered to be crucial to facilitate the development of shared care plans [4]. From a group scale, the chronic care model (CCM) is one of the most studied integrated care models [10-13]. The key to the success of CCM has proved to be the bidirectional communication within multidisciplinary teams and the provision of continuous self-management support to patients [14]. Information technology plays a key role in achieving the above goals, which has been described as a complete eHealth-based feedback loop in the eHealth Enhanced Chronic Care Model (eCCM) [15]. To conclude, information technology is essential to implement effective integrated care for hypertensive patients.

In China, hypertension management is currently delivered based on communities, mainly conducted by 
general practitioners (GPs) [16]. In response to the government policy, physicians and case managers (CMs) are gradually participating in the community-based hypertension management to form a coordinated multidisciplinary team called "three-manager" [17]. Similar to other countries, CMs in China are mainly composed of nurses, working under supervision of GPs [18, 19]. Although the "three-manager" mode has been carried out in some provinces, in practice there still exist some problems. One is that current management guidelines do not clearly specify the responsibility of each role in the "three-manager" mode. Another one is that the unbalanced allocation of medical resources in China brings to the difference in abilities of care providers [20, 21]. GPs and CMs in rural areas can hardly perform comprehensive management following the guidelines. These problems result in a significant gap between standards of care and medical practice in hypertension management $[22,23]$.

\section{Objectives $\{7\}$}

To combat the existing challenges of hypertension management in China, we established a pathwaydriveneHealth-based integrated care model (PEICM) for the "three-manager" mode, focusing on specifying the responsibility of involved roles and providing a standardized and executable care pathway for long-term management. Based on PEICM, a telehealth system has been designed and implemented. This paper describes a twoarm randomized controlled trial to evaluate the effectiveness of PEICM through the use of the system.

\section{Trial design $\{\mathbf{8}\}$}

This 1-year study is designed as a randomized, controlled, and non-blinded superiority trial with two parallel groups and repeated measurements at 3 time points (inclusion, at 6 and 12 months). Randomization will be performed as block randomization with a 1:1 allocation. We used the Additional file 1: SPIRIT checklist [24] and the Additional file 2: TIDieR checklist [25] when writing our manuscript.

\section{Methods: participants, interventions, and \\ outcomes}

\section{Study setting $\{9\}$}

The trial will be conducted in Yibin city, Sichuan province, China. Yibin is a midsize city located in the joint region of Sichuan, Yunnan, and Guizhou province. Given its population, location, economy, and living standard of people, Yibin can represent the central region of China to a certain extent [26]. Therefore, hypertensive patients in Yibin are representative in terms of demographic characteristics and technology acceptance. In this trial, the participating physicians (cardiologists) are recruited from the First People's Hospital of Yibin, which is a tertiary hospital with four major districts (A, B, C, and D). A well-equipped health management center (HMC) is established in $B$ district cooperating with ZICT Technology Co., Ltd. (ZICT). The patient enrolment and outcome measurements will be performed in the HMC. The participating GPs and CMs are from 3 community health centers near the First People's Hospital of Yibin.

\section{Eligibility criteria $\{10\}$}

The inclusion criteria are as follows: (1) 18-80 years old, (2) hypertension diagnosis with uncontrolled blood pressure (BP) for at least 1 year, (3) fluent in Mandarin or Sichuan dialect, (4) able to use the system fluently after instruction, (5) mainly visit the hospital where the trial is hold, and (6) have no walking disability. The exclusion criteria are as follows: (1) participate in another trial within 4 weeks before this study, (2) suffer from mental health problems, (3) suffer from cancer and have received chemotherapy or radiotherapy within half a year before this study, (4) suffer from myocardial infarction, (5) suffer from other serious diseases with natural course shorter than 1 year, (6) have no home internet access, and (7) pregnant or prepare for pregnancy.

\section{Who will take informed consent? $\{26 \mathrm{a}\}$}

All patients eligible for the trial will be invited to HMC in $\mathrm{B}$ district to attend the enrolment session. CMs will apprise them of the information regarding the study and promise that their personal information will only be accessed by the investigators. The patients who agree to participate in the study will be asked to sign informed consent forms before allocation.

\section{Additional consent provisions for collection and use of participant data and biological specimens $\{26 \mathrm{~b}\}$}

The informed consent has included participants' permission to the collection of blood and urine samples, which will be analyzed in the current study. No ancillary study is planned.

\section{Interventions}

Explanation for the choice of comparators $\{6 b$ \}

Management guidelines are considered to be the most convincing evidence to guide the management process. The effectiveness of applying the hypertension management guidelines to primary care in China has been tested [27]. Moreover, the care pathway used in our integrated model is mainly extracted from the guidelines. Therefore, we choose guideline-based management as a usual care intervention for the control group. 


\section{Intervention description $\{11$ a}

Participants in the control group will receive interventions based on the 2018 Chinese Guidelines for Prevention and Treatment of Hypertension [16]. The guidelines emphasize 4 key components of the management in primary care: hierarchical longtermfollow-up, health education, self-management support, and two-way referral. In this trial, a guidelinebased management procedure is as follows: first, patients will be classified into 2 levels according to whether the patients reach target BP, and then, GPs will conduct long-termfollow-up on patients. For level 1 patients, the follow-up frequency is once every 3 months and the follow-up contents focus on complying with current therapy; for level 2 patients, the follow-up frequency is once every 2-4 weeks and the follow-up contents focus on adjusting current therapy to improve BP control. Health education and self-management support will be involved during the follow-up. In addition, a two-way referral channel will be opened to provide further treatment.

Participants in the intervention group will receive interventions based on PEICM, as shown in Fig. 1. The model involves 4 roles in the management: physicians, GPs, CMs, and patients. The responsibility of each role is specified through a well-designed care pathway. The care pathway is initialized based on the guidelines and detailed by several experienced physicians. Nine common tasks are defined in the pathway for hypertension management, which are as follows: diagnosis, risk assessment, hierarchical management, medication guidance, lifestyle guidance, regular follow-up, abnormal condition intervention, health education, and compliance management. The tasks will be generated as executable plans, including doctor intervention plans and patient selfmanagement plans.

The 9 tasks can be grouped into 3 parts: the tasks for regular management, the tasks for pathway variation, and the tasks for self-management. The tasks for regular management include 4 tasks: diagnosis, risk assessment, hierarchical management, and regular follow-up. When a patient is diagnosed with hypertension, he/she will enter the pathway. A cardiovascular risk assessment will firstly be conducted to divide patients into 2 management levels: level 1 for patients in low or middle risk while level 2 for patients in high risk. The management plan for level 1 and level 2 is different. In addition, patients' risk will be reassessed per week based on their self-monitoring data to dynamically change their management levels. Care providers need to perform regular follow-ups for patients, and the follow-up schedule will also be dynamically adjusted based on the management level. Once a patient is in level 2 for over 3 weeks, a two-way referral channel will be opened. The tasks for pathway variation include 2 tasks: abnormal condition intervention and compliance management. When patients' self-monitoring data is abnormal, care providers will immediately be informed and conduct the appropriate intervention; when patients' compliance is low, care providers need to perform an extra follow-up to learn the reason for low compliance and try to improve it. The tasks for self-management include 3 tasks: medication guidance, lifestyle guidance, and health education. Medication guidance aims to provide plans about drug prescription while lifestyle guidance aims to provide plans mainly for diet and physical activity. Health education aims to offer health knowledge to patients, in order to improve their hypertension awareness thus improving their self-management ability.

According to the pathway, the responsibility of each role is specified as follows: physicians are responsible for diagnosis and formulating the self-management plans; GPs are responsible for regular follow-ups, plan adjustment, and abnormal condition intervention; $\mathrm{CMs}$ are responsible for health education and compliance management. Patients need to follow self-management plans and perform self-monitoring to receive timely intervention from care providers.

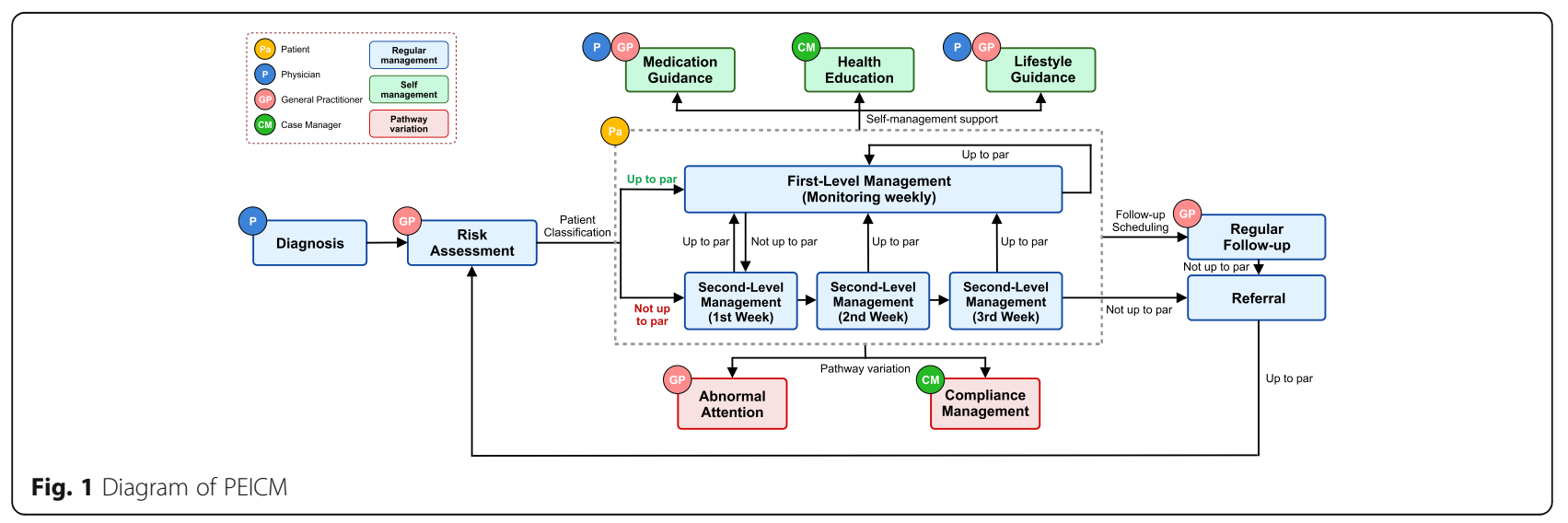


Based on PEICM, a telehealth system has been implemented, which consists of 2 parts: clients for care providers and patients, and a service engine for data storage and pathway execution (Fig. 2). For care providers, the client is in the form of a website that can be accessed on their computers in the hospital. Care providers use the client to supervise patients and conduct the intervention. For patients, the client is in the form of an all-in-one health terminal. The terminal is a combination of an Android tablet with a series of wearable devices (integrating measurement of ECG, heart rate, blood pressure, blood glucose, blood oxygen saturation, and body temperature), which was developed by ZICT (Shenzhen, China). Data measured by the terminal will be automatically uploaded and displayed on the website. In addition, patients can use the tablet to check their self-management plans, read health knowledge, and communicate with care providers. The functional design of the tablet application drew on experience from our previous work [28]. The service engine is a web service deployed on the server, consisting of 4 layers: a data layer, a service layer, a control layer, and an interface layer. The engine will be responsible for saving data from clients and providing decision support based on the pathway.

In this trial, all the interventions will be conducted through the system. The intervention group will gain full access to the system and manage their hypertension under the guidance of the model. The control group will only gain partial access to the system (only for BP measurement) and receive care as usual. Table 1 summarizes all the interventions for each group.

\section{Criteria for discontinuing or modifying allocated interventions $\{11 \mathrm{~b}\}$}

The assigned intervention will only be discontinued in response to participant request. No modification of the interventions is planned during the trial.
Strategies to improve adherence to interventions $\{11 \mathrm{c}\}$ In this trial, adherence to interventions mainly refers to patients' self-management adherence. For the control group, patients will be reminded to follow their selfmanagement regimes during each regular follow-up. For the intervention group, compliance management is defined as a part of the pathway and patient compliance will be calculated by the system per week based on their self-monitoring data. Care providers will not only remind patients in regular follow-ups, but also perform extra follow-ups to improve low adherence.

\section{Relevant concomitant care permitted or prohibited during the trial $\{11 \mathrm{~d}\}$}

No concomitant care or interventions are permitted during the trial.

\section{Provisions for post-trial care $\{30\}$}

This is not applicable. No post-trial care is planned.

\section{Outcomes $\{12\}$}

The primary outcome of the trial is changes in mean systolic blood pressure (SBP) from baseline (T0) to 12 months (T2). The key secondary outcomes are changes in mean diastolic blood pressure (DBP) and changes in biochemical indexes related to hypertension from T0 to T2. The other secondary outcomes include changes in lifestyles, self-management adherence, and hypertension awareness from $\mathrm{T} 0$ to $\mathrm{T} 1$ (6 months) and $\mathrm{T} 2$. In addition, work efficiency of care providers will be assessed at $\mathrm{T} 2$ to validate the model from a care provider perspective.

\section{Participant timeline $\{13\}$}

The SPIRIT figure is presented in Fig. 3, which provides an overview of the schedule of enrolment, interventions, and assessments. Figure 4 shows the timeline diagram.

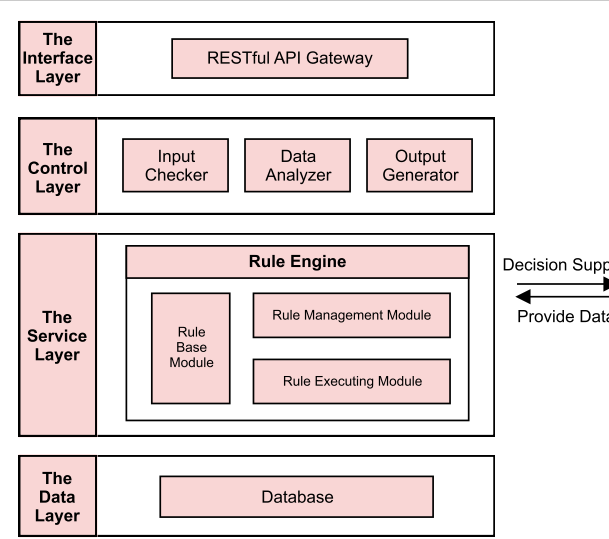

The Service Engine

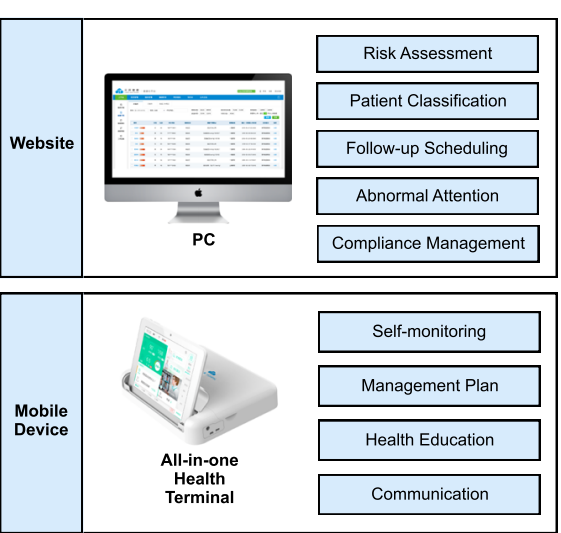

The Clients

Fig. 2 System architecture 
Table 1 All the interventions for the 2 groups

\begin{tabular}{|c|c|c|}
\hline $\begin{array}{l}\text { Intervention } \\
\text { category }\end{array}$ & Execution method in the control group & Execution method in the intervention group \\
\hline Diagnosis & By physicians (before the trial) & By physicians (before the trial) \\
\hline Risk assessment & No execution & $\begin{array}{l}\text { Conducted by GPs with the assistance of the system; } \\
\text { reassessed based on self-monitoring data per week }\end{array}$ \\
\hline $\begin{array}{l}\text { Hierarchical } \\
\text { management }\end{array}$ & $\begin{array}{l}\text { Executed by the system based on patients' BP values; only } \\
\text { adjusted after regular follow-up; no management plans for } \\
\text { patients }\end{array}$ & $\begin{array}{l}\text { Executed by the system based on patients' risk level per } \\
\text { week; provide executable management plans to patients }\end{array}$ \\
\hline $\begin{array}{l}\text { Regular follow- } \\
\text { up }\end{array}$ & $\begin{array}{l}\text { Performed by GPs once every } 3 \text { months for level } 1 \text { patients and } \\
\text { once every } 2-4 \text { weeks for level } 2 \text { patients; no dynamic adjustment }\end{array}$ & $\begin{array}{l}\text { Performed by GPs once every } 3 \text { months for level } 1 \text { patients } \\
\text { and once every } 2 \text { weeks for level } 2 \text { patients; dynamically } \\
\text { adjusted based on patients' management level }\end{array}$ \\
\hline $\begin{array}{l}\text { Abnormal } \\
\text { condition } \\
\text { intervention }\end{array}$ & No execution & $\begin{array}{l}\text { Conducted by GPs; abnormal condition is informed by the } \\
\text { system }\end{array}$ \\
\hline $\begin{array}{l}\text { Compliance } \\
\text { management }\end{array}$ & No execution & $\begin{array}{l}\text { Conducted by CMs; compliance is calculated by the system } \\
\text { per week }\end{array}$ \\
\hline $\begin{array}{l}\text { Medication } \\
\text { guidance }\end{array}$ & Provided by GPs during regular follow-up & Provided by physicians or GPs through the system anytime \\
\hline $\begin{array}{l}\text { Lifestyle } \\
\text { guidance }\end{array}$ & Provided by GPs during regular follow-up & Provided by physicians or GPs through the system anytime \\
\hline $\begin{array}{l}\text { Health } \\
\text { education }\end{array}$ & Provided by GPs during regular follow-up & $\begin{array}{l}\text { Conducted by CMs through the system, mainly in the form o } \\
\text { online materials }\end{array}$ \\
\hline
\end{tabular}

\begin{tabular}{|c|c|c|c|c|}
\hline & \multicolumn{4}{|c|}{ STUDY PERIOD } \\
\hline \multirow[b]{2}{*}{ TIMEPOINT } & Enrolment & Allocation & \multicolumn{2}{|c|}{ Post-allocation } \\
\hline & $-t_{1}$ & $\mathbf{0}$ & $\begin{array}{c}t_{1} \\
\text { (6 months after } \\
\text { allocation) }\end{array}$ & $\begin{array}{c}t_{2} \\
\text { (12 months after } \\
\text { allocation) }\end{array}$ \\
\hline ENROLMENT: & & & & \\
\hline Eligibility screen & $x$ & & & \\
\hline Informed consent & $x$ & & & \\
\hline Allocation & & $x$ & & \\
\hline INTERVENTIONS: & & & & \\
\hline $\begin{array}{r}\text { Usual care } \\
\text { for the control group }\end{array}$ & & & & \\
\hline $\begin{array}{l}\text { PEICM-based care } \\
\text { for the intervention group }\end{array}$ & & & & \\
\hline ASSESSMENTS: & & & & \\
\hline Demographics & $x$ & & & \\
\hline Basic body measurement & $x$ & & $x$ & $\mathrm{X}$ \\
\hline Clinical BP measurement & $x$ & & $x$ & $\mathrm{X}$ \\
\hline Blood test & $x$ & & $x$ & $x$ \\
\hline 24-hour urine test & $x$ & & $x$ & $\mathrm{X}$ \\
\hline $\begin{array}{r}\text { Questionnaire } \\
\text { IPAQ, HB-MS, HK-LS }\end{array}$ & $x$ & & $x$ & $\mathrm{X}$ \\
\hline System log & & & $x$ & $\mathrm{x}$ \\
\hline
\end{tabular}

Fig. 3 SPIRIT figure 


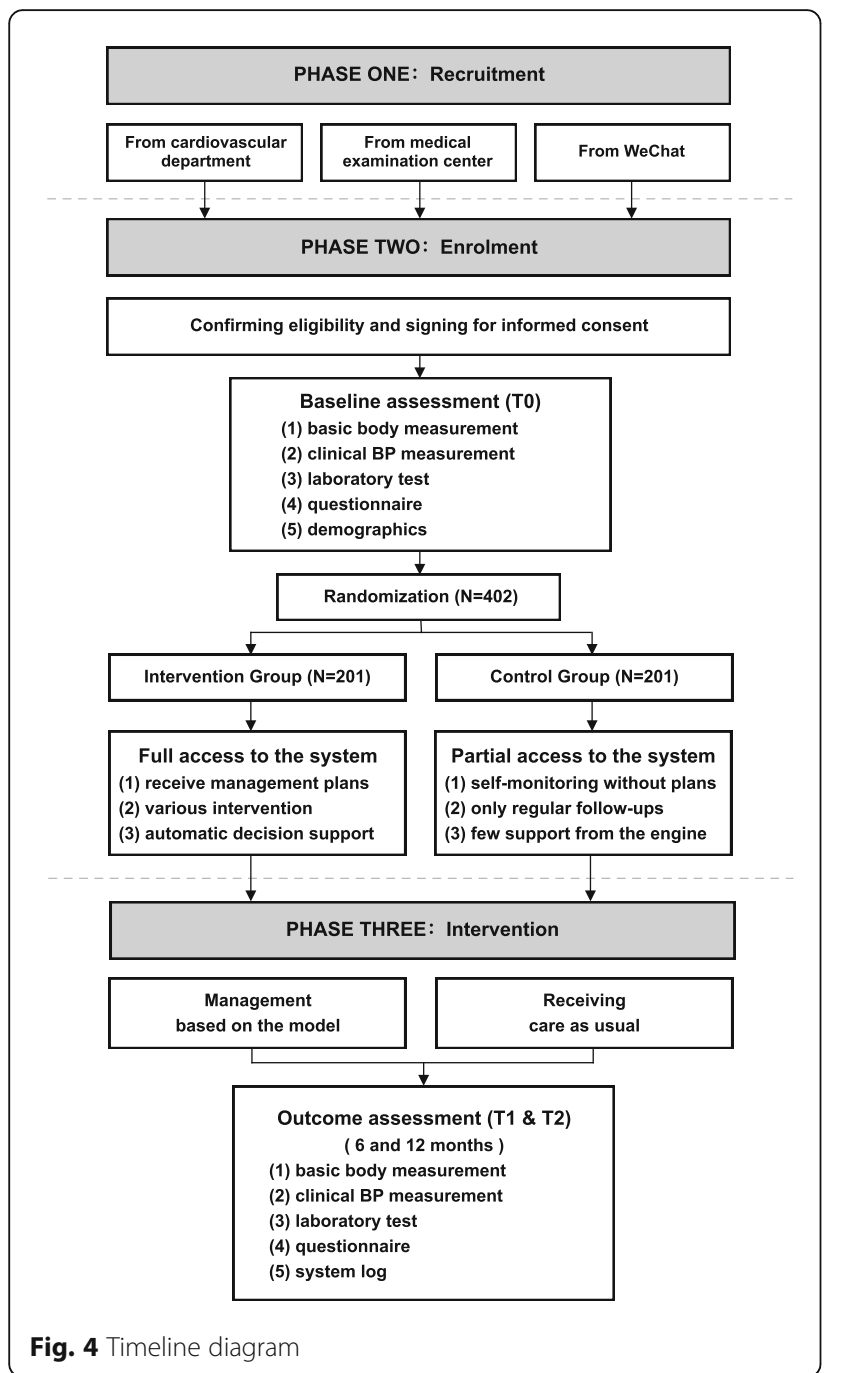

Sample size $\{14\}$

The power calculation is based on the primary outcome measure and aims for a mean systolic blood pressure variation of $5 \mathrm{mmHg}$ with a standard deviation of 16.5 $\mathrm{mmHg}$ (the effect size is based on a previous study [29]). Assuming an $\alpha$ of 5\%, and power of $0.80,171$ patients per group are required at the end of the trial (342 in total). Considering an attrition percentage of $15 \%$, our aim is to include a minimum of 402 patients at baseline.

\section{Recruitment $\{15\}$}

Patients are recruited mainly in 3 ways: (1) review discharged patients' list of the cardiovascular department, (2) review patient list provided by the medical examination center, and (3) recruit patients through physicians' social media (for example, the functional module Moments in WeChat). CMs will call the potentially eligible patients to confirm the eligibility, then invite them to attend the enrolment session in HMC.

\section{Assignment of interventions: allocation}

\section{Sequence generation $\{16 \mathrm{a}\}$}

One investigator will generate the randomized allocation sequence using SPSS version 23.0, which allocates 50\% of the participants to either group.

\section{Concealment mechanism $\{16 \mathrm{~b}\}$}

The allocation sequence is concealed in opaque envelopes from all investigators (except for the investigator who generates the sequence) and care providers until the interventions are assigned.

\section{Implementation $\{16 c\}$}

All patients who give written consent for participation will be enrolled. CMs will guide the enrolment session. First, each patient will be directed to the baseline assessment, including demographics, basic body measurement, clinical BP measurement, laboratory test, and questionnaires. Then, each patient will be randomly assigned an envelope, inside which is a printed random number with the corresponding group. CMs will open the envelope and find the treatment condition to be conducted for this patient. CMs then give the information about treatment allocation to the patient. After that, each group will be guided to different rooms, and CMs will instruct the use of the system to the control group and intervention group, respectively.

\section{Assignment of interventions: blinding} Who will be blinded $\{17 \mathrm{a}\}$

Due to the nature of the intervention, this study is an open-label trial to all participants and care providers. However, in order to reduce the level of bias, the statisticians involved in the data analysis will be blinded to the group allocation.

\section{Procedure for unblinding if needed $\{17 \mathrm{~b}\}$}

This is not applicable. The trial is a non-blinded trial.

\section{Data collection and management}

Plans for assessment and collection of outcomes $\{18 \mathrm{a}\}$

Data collection will be mainly conducted at T0, T1, and T2. Table 2 shows the complete outcome measures and their collecting methods. The details are described in the following sections.

\section{Blood pressure}

Blood pressure (BP) measurement can be categorized into 2 types: clinic and out-of-office BP measurement [16]. Clinic BP measurement is performed by GPs or CMs under standard conditions and unified guidelines. Out-of-office BP measurement includes ambulatory BP monitoring and home BP monitoring, which can provide large amount of BP data outside the medical environment 
Table 2 The complete outcome measures and their collecting methods

\begin{tabular}{|c|c|c|}
\hline Outcome measure & & Collecting method \\
\hline Systolic blood pressure & & Clinic BP measurement \\
\hline Diastolic blood pressure & & Clinic BP measurement \\
\hline \multirow[t]{10}{*}{ Biochemical indexes } & Blood urea nitrogen (BUN) & Laboratory test (blood test) \\
\hline & Creatinine $(\mathrm{Cr})$ & Laboratory test (blood test) \\
\hline & Alanine transaminase (ALT) & Laboratory test (blood test) \\
\hline & Aspartate transaminase (AST) & Laboratory test (blood test) \\
\hline & Uric acid (UA) & Laboratory test (blood test) \\
\hline & Low-density lipoprotein (LDL) & Laboratory test (blood test) \\
\hline & High-density lipoprotein (HDL) & Laboratory test (blood test) \\
\hline & Blood glucose (BG) & Laboratory test (blood test) \\
\hline & Total cholesterol (TC) & Laboratory test (blood test) \\
\hline & Triglyceride (TG) & Laboratory test (blood test) \\
\hline \multirow[t]{3}{*}{ Lifestyles } & Obesity (BMI and WHR) & Basic body measurement \\
\hline & Physical activity & Questionnaire (IPAQ) \\
\hline & Diet (24-h urine sodium and potassium) & Laboratory test (24-h urine test) \\
\hline \multirow[t]{2}{*}{ Self-management adherence } & Medication adherence & Questionnaire (HB-MAS) \\
\hline & Self-monitoring adherence & System log \\
\hline Hypertension awareness & & Questionnaire (HK-LS) \\
\hline \multirow[t]{2}{*}{ Work efficiency of care providers } & Frequency of follow-up in a day & System log \\
\hline & Handling time for a follow-up request & System log \\
\hline
\end{tabular}

$[30,31]$. In this trial, we will use clinic BP measurement to assess the variation in SBP and DBP from baseline to 12 months and use out-of-office BP measurement (home BP monitoring) to promote interventions in daily management. When performing clinic BP measurement, patients should be seated for at least $5 \mathrm{~min}$ in a quiet room before measurements and keep the upper arm at the heart level. Measurements will be conducted at least 2 times and taken 1-2 min apart. An additional measurement will be required in case that the first two readings differ by $>5$ mmHg. The mean value of these readings will be recorded.

\section{Biochemical indexes}

Biochemical indexes are collected from the blood test, as shown in Table 2. These indexes are associated with cardiovascular risk factors, target organ damage, and the corresponding clinical conditions [32], which can indirectly reflect the control of hypertension.

\section{Lifestyles}

Lifestyle measure contains 3 parts: obesity, physical activity, and diet. Obesity is estimated by body mass index (BMI) and waist-to-hip ratio (WHR, for abdominal obesity), which will be measured in the basic body measurement. Physical activity will be assessed by the short form of International Physical Activity Questionnaire
(IPAQ) [33]. Diet is evaluated by the intake of salt. Lowsodium diets will be assessed by the 24-h urine sodium collected from the 24-h urine test. The 24-h measurement of salt excretion in urine has proved to be the best method to measure salt intake [34]. Twenty-fourhour urine potassium will also be considered since increasing potassium intake properly in the diet can help reduce $\mathrm{BP}[35]$.

\section{Self-management adherence}

Adherence to self-management consists of 2 parts: medication adherence and self-monitoring adherence. Medication adherence will be assessed by the Hill-Bone Medication Adherence Scale (HB-MAS) including 9 items [36]. Self-monitoring adherence estimates the compliance with non-pharmacological treatment, which will be assessed by the system log data. We calculate the adherence based on the ratio of actual frequency of BP measurements to the number required by the management plan. The concrete formula can be found in our previous paper [28].

\section{Hypertension awareness}

Patients' hypertension awareness reflects their selfmanagement ability, which will be assessed by the Hypertension Knowledge-Level Scale (HK-LS) [37]. HKLS contains 22 items with 6 sub-dimensions: definition, 
medical treatment, drug compliance, lifestyle, diet, and complication.

\section{Work efficiency of care providers}

Work efficiency of care providers will be calculated based on the follow-up data. In this trial, all the followups are generated, conducted, and recorded through the system. The generated follow-up requests can be divided into 3 types: regular follow-up, abnormal attention follow-up, and compliance follow-up. The system needs to schedule all the follow-ups reasonably to make care providers handle requests timely. We propose 2 indexes to assess the work efficiency: one is the frequency of follow-up in a day, which demonstrates how our system reduces the time cost of a single follow-up; another one is the handling time of a follow-up request, which means the time duration before a generated follow-up request is handled. Intuitively, the more follow-up care providers conduct in a day and the less handling time a follow-up request costs, the more effective their work will be. We will derivate the average work efficiency of care providers in the entire trial period for the control group and the intervention group, respectively. For the handling time of a follow-up request, we will calculate time cost for the 3 types of follow-ups respectively, then sum it together with different weights to get the average time cost.

\section{Plans to promote participant retention and complete follow-up \{18b\}}

At each assessment point ( $\mathrm{T} 1$ and $\mathrm{T} 2$ ), all patients will receive a phone call from $\mathrm{CMs}$ to remind them of the upcoming data collection and free offline consultation with doctors if they attend the assessment. Patients who prematurely discontinue from the study before the 6month evaluation and do not attend any assessment will have the following evaluations performed, if possible: First, GPs will call them to invite them to insist on the trial or attend the assessment; if refused, a brief interview will be conducted to investigate the reasons for discontinuation. Second, the system log data of these patients will be fully explored to extract useful information.

\section{Data management $\{19\}$}

In this trial, most of the data during the management process will be recorded by the system and saved in the database. The assessment data will be entered electronically by an investigator. Double data entry will be performed, and all data ranges will be checked again by a different investigator. All patients, physicians, CMs, and GPs have their unique IDs, and their login passwords are encrypted and kept anonymous to the database administrator. The database is also password protected, with access only to the IPs which have been previously registered in a white list. The investigators are only provided with read permission to the database.

\section{Confidentiality $\{27\}$}

All records that contain personal information will be stored separately in the database. Only care providers can view the information to conduct the intervention. In data analysis, all data will be treated anonymously. Participants' personal information will not be released outside of the study without the written permission.

Plans for collection, laboratory evaluation, and storage of biological specimens for genetic or molecular analysis in this trial/future use $\{33\}$

In this trial, a blood collection and a 24-h urine collection will be carried out in HMC for each participating patient at $\mathrm{T} 0, \mathrm{~T} 1$, and $\mathrm{T} 2$. The blood and urine samples will be used for laboratory tests to assess the biochemical indexes related to hypertension (see Table 2). The test results will be sent to investigators and all samples will not be stored. Investigators will not have access to personal identifiers.

\section{Statistical methods}

Statistical methods for primary and secondary outcomes $\{20 a\}$

Statistical analysis will be performed using SPSS version 23.0. All statistical tests will be reported as a two-sided significance level of $5 \%$, and the results will be presented with two-sided 95\% confidence intervals (CIs). The data will be treated either by parametric or non-parametric analysis based on the tests for normal distribution (Shapiro-Wilk test) and homoscedasticity (Levene's test). We will first conduct detailed exploratory descriptive analyses to collected data using graphical and numerical summaries. Then, comparisons between and within groups will be performed to identify the effectiveness. For categorical variables, the Pearson chi-square test or Fisher exact test will be used; for continuous variables, the Student $t$ test or Mann-Whitney test will be used. In case of significant baseline differences, a linear mixed model will be constructed for the outcomes adjusting the baseline using the following covariates as appropriate: baseline SBP, demographics (age, gender, education levels), and BMI. Only those confounding factors found to be statistically significant to the outcomes by analysis of covariance will be included in the final model.

\section{Interim analyses $\{21 b\}$}

This is not applicable. No formal interim analysis of the primary and secondary outcomes is planned. 
Methods for additional analyses (e.g., subgroup analyses) \{20b\}

We will also perform subgroup analyses of the primary and secondary outcomes stratified by following variates at the initial management (1 week after baseline): BMI $(<24$, $\geq 24)$, management level (level 1 and level 2), and self-management adherence.

Methods in analysis to handle protocol non-adherence and any statistical methods to handle missing data $\{20 \mathrm{c}\}$ The statistical analysis will be conducted on an intention-to-treat (ITT) basis. For incomplete assessments (missing data), multiple imputation will be applied using both an optimistic and pessimistic scenario.

Plans to give access to the full protocol, participant-level data, and statistical code $\{31 \mathrm{c}\}$

The participant-level dataset and statistical code generated during the current study will be available from the corresponding author on reasonable request after the trial is finished.

\section{Oversight and monitoring}

Composition of the coordinating center and trial steering committee $\{5 \mathrm{~d}\}$

Two committees have been established to manage the trial, including a trial steering committee and a data monitoring committee. The steering committee composes of cardiologists from the First People's Hospital of Yibin and investigators from Zhejiang University. The major responsibility of the steering committee is to evaluate and study protocol, communicate with the ethics committee, and supervise the practice of hypertension management for both 2 groups.

\section{Composition of the data monitoring committee, its role and reporting structure $\{21 \mathrm{a}\}$}

The data monitoring committee consists of statisticians from Zhejiang University. This committee is independent from the sponsor and has no competing interests. The committee will be in charge of auditing the trial every quarter to oversee the data quality of the study. The committee charter is available from the corresponding author.

\section{Adverse event reporting and harms $\{22\}$}

Serious adverse events will be collected and recorded for further analysis at the end of the trial. These events include deaths, emergency room visits, and hospitalization longer than $24 \mathrm{~h}$. Investigators will explore the correlation between these events and patients' healthcare records.
Frequency and plans for auditing trial conduct $\{23\}$

The trial conduct will be audited by the data monitoring committee once every quarter.

\section{Plans for communicating important protocol amendments to relevant parties (e.g., trial participants, ethical committees) $\{25\}$}

All changes to the study protocol will be reviewed by the steering committee, then reported to the sponsor, participating care providers, and investigators.

\section{Dissemination plans $\{31$ a}

The study results will be disseminated via articles published in peer-reviewed journals.

\section{Discussion}

This protocol has been designed to understand the effectiveness of a pathway-driveneHealth-based integrated care model, implemented as a full-featured telehealth system. Compared with prior studies [38, 39], our study innovatively introduces the concept of pathway, then combines it with information technology and the "three-manager" mode to establish a standardized and executable care model. Besides testing efficacy of the model, this study will have several other strengths if successful. First, the model can be generalized to other common chronic diseases, such as diabetes, stroke, and chronic obstructive pulmonary disease (COPD). The task-based pathway can be applied in both single disease and multiple chronic conditions. Second, the study would provide the feasibility evidence of the "three-manager" mode combined with information technology in China. Policy makers in other regions across the country could roll out similar services for better management. Last, the awareness, treatment, and control rates of hypertension in Sichuan, Yunnan, and Guizhou are relatively low compared with other provinces [40]. Therefore, performing such a trial in Yibin is significant to improve the local outcome for hypertension management.

The present protocol is also subject to some limitations. First, since the clients for patients are different from traditional mobile applications, participants may show different levels of acceptance in the system. Low system acceptance will lead to undesirable high attrition rates. We will adopt several strategies to improve patients' acceptance of the system, including ongoing system updates with usability tests, online system guidance from CMs, and regular offline training sessions for patients. Second, this is an openlabel trial for both patients and care providers. Patients in the control group may not comply with selfmanagement regimes or even choose to discontinue the participation, and care providers may tend to conduct 
the management for the control group not following the guidelines. We plan to eliminate these potential biases through intensive training of care providers and reward mechanism on patients (for example, free outpatient registration), as well as supervision of intervention quality from the steering committee.

\section{Trial status}

This study protocol was approved on 7 November 2019, and this manuscript details the protocol on the original version. Due to the COVID-19 pandemic, the participant recruitment has been adjusted to July 2020. The first participant was enrolled on July 3, 2020, and recruitment is expected to be completed by November 2020 .

\section{Supplementary Information}

The online version contains supplementary material available at https://doi. org/10.1186/s13063-021-05020-2.

\section{Additional file 1. SPIRIT Checklist.}

Additional file 2. TIDieR checklist.

\section{Abbreviations}

BP: Blood pressure; CMs: Case managers; GPs: General practitioners; HBMAS: The Hill-Bone Medication Adherence Scale; HK-LS: The Hypertension Knowledge-Level Scale; IPAQ: The International Physical Activity Questionnaire; PEICM: Pathway-driven eHealth-based integrated care model

\section{Acknowledgements}

We want to thank Professor Ping Yu and Professor Leonard Arnolda from University of Wollongong for their valuable suggestions on the trial design.

\section{Authors' contributions $\{31 \mathrm{~b}\}$}

ZW and $C L$ designed the model. ZW, JA, WH, and $L H$ developed the system. $Z W, Y C$, and $C L$ designed the trial. DW and ZW collected and designed the questionnaires. YL, MZ, YC, ZW, and LW assisted in the participant recruitment. $Z W$ drafted the manuscript. $H D$ and ND made critical revisions to the manuscript. All authors read and approved the final manuscript.

\section{Competing interests $\{28\}$}

The authors declare that they have no competing interests.

\section{Funding $\{4\}$}

This study was supported by the National Key Research and Development Program of China (No. 2020YFC2006400, No. 2017YFC0114105, and No. 2017YFB1002301), the key Research and Development Program of Zhejiang Province of China (No. 2021C03111), the Key Research and Development Program of Guangxi Zhuang Autonomous of China (No. 2020AB33002), and the Key Research and Development Program of Ningxia Hui Autonomous of China (No. 2018BFG02009). The funding sources have no role in the design, conduct, or reporting of the study.

\section{Availability of data and materials $\{29\}$}

The datasets generated and/or analyzed during the current study are not publicly available due to individual privacy but are available from the corresponding author on reasonable request.

\section{Consent for publication $\{32\}$}

This consent form is part of the informed consent process, which is available from the corresponding author on request.

Ethics approval and consent to participate $\{24\}$

This study was approved by the Medical Ethics Committee of the First People's Hospital of Yibin (Approved Number 2019-005). All participants have provided written informed consent.

\section{Author details}

${ }^{1}$ College of Biomedical Engineering and Instrument Science, The Ministry of Education Key Laboratory of Biomedical Engineering, Zhejiang University, Hangzhou 310027, China. ${ }^{2}$ The First People's Hospital of Yibin, Yibin, China. ${ }^{3}$ ZICT Technology Co.,Ltd., Shenzhen, China.

Received: 7 February 2020 Accepted: 4 January 2021

Published online: 22 January 2021

\section{References}

1. Wang Z, Chen Z, Zhang L, Wang X, Hao G, Zhang Z, et al. Status of hypertension in China. Circulation. 2018;137(22):2344-56. https://doi.org/10. 1161/CIRCULATIONAHA.117.032380.

2. Reynolds R, Dennis S, Hasan I, Slewa J, Chen W, Tian D, et al. A systematic review of chronic disease management interventions in primary care. BMC Fam Pract. 2018;19(1):11. https://doi.org/10.1186/s12875-017-0692-3.

3. Nguyen Q, Dominguez J, Nguyen L, Gullapalli N. Hypertension management: an update. Am Heal Drug Benefits. 2010;3(1):47-56 Available from: http://www.ncbi.nlm.nih.gov/pubmed/25126308.

4. WHO Regional Office for Europe. Integrated care models: an overview [Internet]. 2016. available from: https://www.euro.who.int/_data/assets/ pdf_file/0005/322475/Integrated-care-models-overview.pdf. Accessed 18 Jan 2020.

5. WHO Regional Office for Europe. Strengthening people-centred health systems in the WHO European Region: framework for action on integrated health services delivery [Internet]. 2016. available from: https://www.euro. who.int/_data/assets/pdf_file/0004/315787/66wd15e_FFA_IHSD_160535. pdf. Accessed 18 Jan 2020

6. Hamine S, Gerth-Guyette E, Faulx D, Green BB, Ginsburg AS. Impact of mHealth chronic disease management on treatment adherence and patient outcomes: a systematic review. J Med Internet Res. 2015;17(2):e52. https:// doi.org/10.2196/jmir.3951.

7. Kumar N, Khunger M, Gupta A, Garg N. A content analysis of smartphonebased applications for hypertension management. J Am Soc Hypertens. 2015;9(2):130-6. https://doi.org/10.1016/j.jash.2014.12.001.

8. Harrison CE, Wild K. Using telehealth in the management of hypertension. Nurs Stand. 2017:31(48):44-9. https://doi.org/10.7748/ns.2017.e10618.

9. Curry N, Ham C. Clinical and service integration: the route to improved outcomes. [Internet]. The King's Fund; 2010. available from: https://www. kingsfund.org.uk/sites/files/kf/Clinical-and-service-integration-Natasha-CurryChris-Ham-22-November-2010.pdf. Accessed 19 Jan 2020.

10. Wagner EH, Austin BT, Von Korff M. Organizing care for patients with chronic illness. Milbank Q. 1996;74(4):511-44 Available from: http://www. ncbi.nlm.nih.gov/pubmed/8941260.

11. Wagner EH, Austin BT, Davis C, Hindmarsh M, Schaefer J, Bonomi A. Improving chronic illness care: translating evidence into action. Health Aff. 2001;20(6):64-78. https://doi.org/10.1377/hlthaff.20.6.64.

12. Bodenheimer T, Wagner EH, Grumbach K. Improving primary care for patients with chronic illness. JAMA. 2002;288(14):1775-9 Available from: http://www.ncbi.n/m.nih.gov/pubmed/12365965.

13. Bodenheimer T, Wagner EH, Grumbach K. Improving primary care for patients with chronic illness: the chronic care model, Part 2. JAMA. 2002; 288(15):1909-14 Available from: http://www.ncbi.nlm.nih.gov/pubmed/123 77092.

14. Coleman K, Austin BT, Brach C, Wagner EH. Evidence on the Chronic Care Model in the new millennium. Health Aff (Millwood). 2009;28(1):75-85. https://doi.org/10.1377/hlthaff.28.1.75.

15. Gee PM, Greenwood DA, Paterniti DA, Ward D, Miller LMS. The eHealth Enhanced Chronic Care Model: a theory derivation approach. J Med Internet Res. 2015;17(4):e86. https://doi.org/10.2196/jmir.4067.

16. Joint Committee for Guideline Revision. 2018 Chinese Guidelines for Prevention and Treatment of Hypertension-a report of the Revision Committee of Chinese Guidelines for Prevention and Treatment of Hypertension. J Geriatr Cardiol. 2019;16(3):182-241. https://doi.org/10.11909/ j.issn.1671-5411.2019.03.014.

17. Yang S. Introduction of general practitioner education and chronic disease management model in Xiamen. Chinese Gen Pract. 2017;20(202):2526-7. https://doi.org/10.3969/j.issn.1007-9572.2017.20.020.

18. Bourqueil Y, Marek A, Mousques J. Practice, role and position of nurses in primary care in six European countries, in Ontario and in Quebec. Rech 
Soins Infirm. 2008;(93):94-105 Available from: http://www.ncbi.nIm.nih.gov/ pubmed/18678084. Accessed 1 Feb 2020.

19. van Dillen SME, Hiddink GJ. To what extent do primary care practice nurses act as case managers lifestyle counselling regarding weight management? A systematic review. BMC Fam Pract. 2014;15:197. https://doi.org/10.1186/ s12875-014-0197-2.

20. Feng $X L$, Pang M, Beard J. Health system strengthening and hypertension awareness, treatment and control: data from the China Health and Retirement Longitudinal Study. Bull World Health Organ. 2014;92(1):29-41. https://doi.org/10.2471/BLT.13.124495.

21. Hernandez J, Anderson S. Storied experiences of nurse practitioners managing prehypertension in primary care. J Am Acad Nurse Pract. 2012; 24(2):89-96. https://doi.org/10.1111/j.1745-7599.2011.00663.x.

22. Li H. Hypertension management in primary care in China: still a long way to proceed. J Gen Pract. 2015;04(02):2-3. https://doi.org/10.4172/2329-9126. 1000238.

23. Li H, Wei X, Wong MC, Yang N, Wong SY, Lao X, et al. A comparison of the quality of hypertension management in primary care between Shanghai and Shenzhen: a cohort study of 3196 patients. Medicine (Baltimore). 2015, 94(5):e455. https://doi.org/10.1097/MD.0000000000000455.

24. Chan A-W, Tetzlaff JM, Altman DG, Laupacis A, Gøtzsche PC, Krleža-Jerić K, et al. SPIRIT 2013 statement: defining standard protocol items for clinical trials. Ann Intern Med. 2013;158(3):200-7. https://doi.org/10.7326/0003-4819158-3-201302050-00583.

25. Hoffmann TC, Glasziou PP, Boutron I, Milne R, Perera R, Moher D, et al. Better reporting of interventions: template for intervention description and replication (TIDieR) checklist and guide. BMJ. 2014;348:g1687. https://doi. org/10.1136/bmj.g1687.

26. National Bureau of Statistics. China city statistical yearbook 2017 [Internet]. China Statistics Press; 2017. available from: http://www.stats.gov.cn/tjjj/ ndsj/2017/indexeh.htm. Accessed 3 Feb 2020.

27. Wang X, Li W, Li X, An N, Chen H, Jan S, et al. Effects and cost-effectiveness of a guideline-oriented primary healthcare hypertension management program in Beijing, China: results from a 1-year controlled trial. Hypertens Res. 2013;36(4):313-21. https://doi.org/10.1038/hr.2012.173.

28. Duan H, Wang Z, Ji Y, Ma L, Liu F, Chi M, et al. Using goal-directed design to create a mobile health app to improve patient compliance with hypertension self-management: development and deployment. JMIR mHealth uHealth. 2020;8(2):e14466. https://doi.org/10.2196/14466.

29. McManus RJ, Mant J, Bray EP, Holder R, Jones MI, Greenfield S, et al. Telemonitoring and self-management in the control of hypertension (TASM INH2): a randomised controlled trial. Lancet. 2010;376(9736):163-72. https:// doi.org/10.1016/S0140-6736(10)60964-6.

30. Dolan E, Stanton A, Thijs L, Hinedi K, Atkins N, McClory S, et al. Superiority of ambulatory over clinic blood pressure measurement in predicting mortality: the Dublin outcome study. Hypertens (Dallas, Tex 1979). 2005;46(1):156-61. https://doi.org/10.1161/01.HYP.0000170138.56903.7a.

31. Niiranen TJ, Hänninen M-R, Johansson J, Reunanen A, Jula AM. Homemeasured blood pressure is a stronger predictor of cardiovascular risk than office blood pressure: the Finn-Home study. Hypertens (Dallas, Tex 1979). 2010:55(6):1346-51. https://doi.org/10.1161/HYPERTENSIONAHA.109.149336.

32. O'Shea PM, Griffin TP, Fitzgibbon M. Hypertension: The role of biochemistry in the diagnosis and management. Clin Chim Acta 2017:465:131-143. doi: https://doi.org/10.1016/j.cca.2016.12.014.

33. Craig $C L$, Marshall AL, Sjöström M, Bauman AE, Booth ML, Ainsworth $B E$, et al. International physical activity questionnaire: 12-country reliability and validity. Med Sci Sports Exerc. 2003;35(8):1381-95. https://doi.org/10.1249/01. MSS.0000078924.61453.FB.

34. McLean RM. Measuring population sodium intake: a review of methods. Nutrients. 2014;6(11):4651-62. https://doi.org/10.3390/nu6114651.

35. Aburto NJ, Hanson S, Gutierrez H, Hooper L, Elliott P, Cappuccio FP. Effect of increased potassium intake on cardiovascular risk factors and disease: systematic review and meta-analyses. BMJ. 2013;346(apr03 3):f1378. https:// doi.org/10.1136/bmj.f1378.

36. Kim MT, Hill MN, Bone LR, Levine DM. Development and testing of the HillBone Compliance to High Blood Pressure Therapy Scale. Prog Cardiovasc Nurs. 2000;15(3):90-6. https://doi.org/10.1111/j.1751-7117.2000.tb00211.x.

37. Erkoc SB, Isikli B, Metintas S, Kalyoncu C. Hypertension Knowledge-Level Scale (HK-LS): a study on development, validity and reliability. Int J Environ Res Public Health. 2012;9(3):1018-29. https://doi.org/10.3390/ijerph9031018.
38. Xu L, Fang W-Y, Zhu F, Zhang H-G, Liu K. A coordinated PCP-Cardiologist Telemedicine Model (PCTM) in China's community hypertension care: study protocol for a randomized controlled trial. Trials. 2017;18(1):236. https://doi. org/10.1186/s13063-017-1970-z.

39. Tu Q, Xiao LD, Ullah S, Fuller J, Du H. Hypertension management for community-dwelling older people with diabetes in Nanchang, China: study protocol for a cluster randomized controlled trial. Trials. 2018;19(1):385. https://doi.org/10.1186/s13063-018-2766-5.

40. Yin M, Augustin B, Fu Z, Yan M, Fu A, Yin P. Geographic distributions in hypertension diagnosis, measurement, prevalence, awareness, treatment and control rates among middle-aged and older adults in China. Sci Rep. 2016;6(1):37020. https://doi.org/10.1038/srep37020.

\section{Publisher's Note}

Springer Nature remains neutral with regard to jurisdictional claims in published maps and institutional affiliations.
Ready to submit your research? Choose BMC and benefit from:

- fast, convenient online submission

- thorough peer review by experienced researchers in your field

- rapid publication on acceptance

- support for research data, including large and complex data types

- gold Open Access which fosters wider collaboration and increased citations

- maximum visibility for your research: over $100 \mathrm{M}$ website views per year

At BMC, research is always in progress.

Learn more biomedcentral.com/submissions 